\title{
Myopathy following postoperative ablative radioiodine for follicular carcinoma of the thyroid
}

\author{
Hermione C Price \\ Vijay Jayagopal \\ Centre for Diabetes and \\ Endocrinology, York Hospital, \\ York, UK
}

Correspondence: Vijay Jayagopal Centre for Diabetes and Endocrinology, York Hospital,York YO3I 8HE UK Tel +44 I904 72II 75

Email vijay.jayagopal@york.nhs.uk

\begin{abstract}
We highlight a case of disabling myopathy following radioablative iodine treatment for follicular carcinoma of the thyroid. A 34-year-old man presented with a tender neck swelling, ultrasound and biopsy were suggestive of thyroid malignancy. Thyroidectomy was undertaken and histology confirmed follicular carcinoma of the thyroid. Treatment with ablative radioiodine followed and within days the patient developed disabling myopathy. Investigations to date do not reveal any other cause for the myopathy and there is no evidence to suggest that this is a paraneoplastic phenomenon. We believe this is the first reported case of ablative radioiodine-induced mypoathy.
\end{abstract}

Keywords: thyroid neoplasms, muscular diseases, iodine radioisotopes

\section{Introduction}

Whilst neuromuscular symptoms are widely reported in both hypothyroidism and hyperthyroidism, the same cannot be said of thyroid carcinoma or following ablative radioiodine therapy. We present a unique case of disabling myopathy following radioablative iodine treatment for follicular carcinoma of the thyroid.

\section{Case report}

A 34-year-old man presented to the ear, nose, and throat department with a one-day history of a tender swelling in the neck. He had no significant past medical history and was otherwise well. Examination confirmed a swelling in the right lobe of the thyroid. Subsequent thyroid ultrasound and tru-cut biopsy were suggestive of follicular carcinoma of the thyroid. He underwent right thyroid lobectomy. Histology confirmed follicular carcinoma and a completion thyroidectomy was performed preserving the left parathyroid glands. Histology from the completion thyroidectomy showed no further neoplasia and none of the twelve lymph nodes removed showed evidence of local metastases. Replacement therapy with tri-iodothyronine was commenced immediately postoperatively and this was continued until two weeks prior to undergoing ablative radioiodine therapy. Within a few days of receiving oral ablative radioiodine at a dose of $3.7 \mathrm{GBq}$, he developed myalgia.

The myalgia was described as a constant ache particularly involving the limb girdles and proximal limb and trunk muscles. Symptoms worsened after exercise. The patient also reported myokimic rippling in most muscle groups. The myalgia was such that his sleep was impaired. He admitted anxiety and reported an improvement after a period of cognitive behavior therapy. He denied personal stress otherwise, lived with his wife and 4-year-old child and continued working as an information technology manager. He received amitriptyline and later gabapentin which helped him sleep better, but did not resolve his myalgia.

The myalgia has persisted for five years postablative radioiodine therapy. It has continued after thyroid-stimulating hormone (TSH) suppression and his symptoms do not change with variation in his TSH levels. 
Examination did not reveal any signs of peripheral nervous system, neuromuscular junction, or myopathic disorder. No myokimic movements or fasciculations were seen. Investigations were undertaken in order to investigate the cause of his myalgia. Nerve conduction studies were normal and needle electromyelography did not demonstrate any neurogenic or myopathic features or features of membrane instability or myokimic or cramp discharges. Biochemistry showed a modestly elevated creatinine phosphokinase of $279 \mathrm{U} / \mathrm{KL}$. Urea and electrolytes, calcium, magnesium, and phosphate were all within the normal range. Parathyroid hormone levels were also within the normal range. Fasting glucose was $81 \mathrm{mg} / \mathrm{dl}$. Ferritin, B12, and folate were also within normal limits. Erythrocyte sedimentation rate was $4 \mathrm{~mm} /$ hour (normal range $1-10 \mathrm{~mm} /$ hour). A short synacthen test was normal and random cortisol was $207 \mathrm{nmol} / \mathrm{l}$. Chest radiograph was normal. Thyroid peroxidase antibody was not detectable. Thyroid biochemistry showed free thyroxine $30 \mathrm{pmol} / \mathrm{L}$ and $\mathrm{TSH}<0.01 \mathrm{mU} / \mathrm{L}$. Thyroglobulin remained undetectable and a radioiodine uptake scan performed off levothyroxine did not show any residual thyroid tissue. There was no history of HMG-CoA reductase inhibitor use or alcohol abuse.

\section{Discussion}

Acquired myopathies are most commonly caused by inflammatory disorders including dermatomyositis and connective tissue disorders, toxins including drugs and alcohol, and endocrine disorders including thyroid disease, Cushing's syndrome and Addison's disease. ${ }^{1}$ Other causes include neuromuscular junction disorders such as myasthenia gravis, secondary to infection, including HIV and AIDS, and as a paraneoplastic phenomenon. ${ }^{1}$ The patient in this case had no clinical features suggestive of an inflammatory disorder and erythrocyte sedimentation rate was normal. There was no history of alcohol abuse or use of HMG-CoA reductase inhibitor drugs or corticosteroids. A short synacthen test excluded Addison's disease and a random cortisol was not elevated. Clinical features were also not in keeping with a diagnosis of Cushing's syndrome. The commonest paraneoplastic myopathy is Lambert Eaton syndrome seen with carcinoma of the bronchus. Chest radiograph did not show evidence of bronchial malignancy. There is no history of HIV or AIDS or risk factors for these conditions.

The improvement in symptoms with cognitive behavior therapy has been reported previously in individuals with chronic muscular pain. ${ }^{2}$ Efficacy is thought to be related to the listening, understanding, acceptance, tolerance, and confirmation provided by the therapist. ${ }^{2}$
Radiation myelopathy can occur months or years after exposure to radiation. It is characterized by demyelination, loss of axons and focal necrosis and no treatment has been found to be of conclusive benefit. ${ }^{3}$ Symptoms must be experienced within the segment of spinal cord exposed to radiation. The rapid onset and widespread symptoms in this case make this a distinct condition from radiation myelopathy.

Individuals with thyroid dysfunction commonly report neuromuscular symptoms. Up to $80 \%$ of those with hypothyroidism report such symptoms. ${ }^{4}$ The commonest complaints are of muscle weakness, fatiguability, pain, stiffness, or cramps. Evidence of weakness is found on clinical examination in one third of patients and one third also have myopathic changes on electrophysiological examination. Fewer than $20 \%$ have evidence of decreased nerve conduction velocity or fibrillation potentials. Neuromuscular symptoms resolve in the majority of patients once treatment with levothyoxine is initiated with only a minority reporting weakness after one year.

In hyperthyroidism, two thirds of patients report muscle weakness and $10 \%$ fatigability, muscle pain or cramps. Clinical examination confirms reduced muscle strength in two thirds and $10 \%$ have myopathic and a quarter neuropathic changes in electrophysiology. Symptoms largely resolve within three months of treatment. ${ }^{4} \mathrm{~A}$ small number of patients with thyrotoxicosis, usually Asian men, develop thyrotoxic periodic paralysis, but this resolves with treatment of the underlying thyrotoxicosis. ${ }^{5}$

Changes in skeletal muscle expression of myosin-heavychain genes have been reported to occur in thyroid disease states. ${ }^{6}$ It has been postulated that the common discrepancy between clinical and electrophysiological findings could be because phenotypic differences may not necessarily alter electromyographic studies.

Thyroid malignancies most often present as a lump in the neck. Diagnosis is made by fine needle aspiration cytology and total thyroidectomy follows. ${ }^{7}$ Thyroid replacement therapy is commenced in the immediate postoperative period. This is continued until approximately two weeks prior to the administration of radioiodine ablation. During this period the patient is rendered hypothyroid to increase levels of TSH and improve the outcome of radioiodine ablation. Adjuvant radioiodine ablation of residual thyroid tissue following thyroidectomy is given to destroy any microscopic foci of disease, to increase the specificity of subsequent 131I scanning for detection of recurrent or metastatic disease ${ }^{7}$ and to improve the value of measurements of serum thyroglobulin as a serum marker derived only from 
malignant thyroid cells. Combined retrospective data show radioiodine ablation reduces long-term disease-specific mortality in patients with primary tumors $>1 \mathrm{~cm}$ in diameter, multicentric tumors or those with soft tissue invasion at presentation ${ }^{7}$ and this is reflected in current guidelines. $^{8}$

Reported short-term complications of radioiodine ablation include radiation thyroiditis, painless neck edema, sialoadenitis, tumor hemorrhage or edema, and rarely pulmonary fibrosis after treatment of diffuse pulmonary metastases. Even rarer still are vocal cord paralysis ${ }^{9}$ and airway obstruction. ${ }^{10}$ The occurrence of short-term adverse events appears to be dose-related. ${ }^{11}$ Longer-term complications include an increased frequency of malignant diseases including acute myeloid leukemia, bladder, salivary gland, colon and breast carcinoma.

It is possible that in the case reported here, myopathy could have been provoked by the period of hypothyroidism following withdrawal of thyroid replacement therapy prior to radioiodine ablation. This seems unlikely given the temporal relationship between the administration of radioiodine and the development of symptoms and the duration of symptoms. The abnormal accumulation of glycogen in muscle fibers that occurs with hypothyroidism-induced myopathy has been shown to improve once thyroid replacement therapy is initiated and normal serum concentrations of thyroid hormones restored. ${ }^{6,12}$ This may take up to six months because of the inherent latency of the action of thyroid hormones on the cell nucleus, ${ }^{6}$ but is unlikely to persist chronically over several years as described here.
In conclusion, we report a patient with a previously unreported disabling myopathy following postoperative ablative radioiodine therapy for follicular carcinoma of the thyroid in the absence of any well recognized cause of myopathy.

\section{Disclosure}

The authors report no conflicts of interest in this work.

\section{References}

1. Hilton-Jones D. Myopathies. Rev Clin Gerontol. 2001;11:131-147.

2. Steihaug S, Ahlsen B, Malterud K. "I am allowed to be myself": women with chronic muscular pain being recognized. Scand J Public Health. 2002;30(4):281-287.

3. Rampling R, Symonds P. Radiation myelopathy. Curr Opin Neurol. 1998;11(6):627-632.

4. Duyff R, Bosch JVd, Laman D, Loon BPv, Wm HL. Neuromuscular findings in thyroid dysfunction: a prospective clinical and electrodiagnostic study. J Neurol Neurosurg Psychiatry. 2000;68:750-755.

5. Darrow M, Brammer W, Rowley A. Thyrotoxic periodic paralysis: Two case studies. Arch Phys Med Rehabil. 1995;76(7):685-687.

6. Klein I, Ojamaa K. Thyroid (neuro) myopathy. Lancet. 2000;356: 614-615.

7. Sherman-Steven I. Thyroid carcinoma. Lancet. 2003;361:501-511.

8. Thyroid cancer guidelines development group, the British Thyroid Association, the Royal College Of Physicians. Guidelines for the management of thyroid cancer. 2nd edition. London, UK: British Thyroid Association, Royal College of Physicians; 2007.

9. Beshyah S, Al-Fallouji H, Neave F. Complete right vocal cord paralysis: a very rare complication of radioiodine therapy for hyperthyroidism. Endocrine Abstracts. 2004;7:P. 301.

10. Kinuya S, Yoneyama T, Michigishi T. Airway complication occurring during radioiodine treatment for Graves' disease. Ann Nucl Med. 2007;21(6):367-369.

11. Lin W, Shen Y, Wang S. Short-term hazards of low-dose radioiodine ablation therapy in postsurgical thyroid cancer patients. Clin Nucl Med. 1996;21(10):780-782.

12. Lin R, Liu C, Tai C, Lai C. Hypothyroid myopathy-pathological and ultrastructural study. Kaohsiung J Med Sci. 2000;16(2):68-75. 
